\title{
Effect of Meissner Screening and Trapped Magnetic Flux on Magnetization Dynamics in Thick $\mathrm{Nb} / \mathrm{Ni}_{80} \mathrm{Fe}_{20} / \mathrm{Nb}$ Trilayers
}

\author{
Kun-Rok Jeon, $, 1,2,{ }^{*}$ Chiara Ciccarelli, ${ }^{2}$ Hidekazu Kurebayashi, ${ }^{3}$ Lesley F. Cohen, ${ }^{4}$ Xavier Montiel, ${ }^{5}$ \\ Matthias Eschrig, ${ }^{5}$ Thomas Wagner, ${ }^{1,6}$ Sachio Komori, ${ }^{1}$ Anand Srivastava, ${ }^{1}$ Jason W.A. Robinson, ${ }^{1}$ and \\ Mark G. Blamire ${ }^{1}$ \\ ${ }^{1}$ Department of Materials Science and Metallurgy, University of Cambridge, 27 Charles Babbage Road, \\ Cambridge CB3 OFS, United Kingdom \\ ${ }^{2}$ Cavendish Laboratory, University of Cambridge, Cambridge CB3 OHE, United Kingdom \\ ${ }^{3}$ London Centre for Nanotechnology and Department of Electronic and Electrical Engineering at University of \\ College London, London WC1H 01H, United Kingdom \\ ${ }^{4}$ The Blackett Laboratory, Imperial College London, SW7 2AZ, United Kingdom \\ ${ }^{5}$ Department of Physics, Royal Holloway, University of London, Egham Hill, Egham, Surrey TW20 OEX, \\ United Kingdom \\ ${ }^{6}$ Hitachi Cambridge Laboratory, Cambridge CB3 OHE, United Kingdom
}

(Received 18 October 2018; published 30 January 2019)

\begin{abstract}
We investigate the influence of Meissner screening and trapped magnetic flux on magnetization dynamics for a $\mathrm{Ni}_{80} \mathrm{Fe}_{20}$ film sandwiched between two thick Nb layers $(100 \mathrm{~nm})$ using broadband $(5-20 \mathrm{GHz})$ ferromagnetic resonance (FMR) spectroscopy. Below the superconducting transition $T_{c}$ of $\mathrm{Nb}$, significant zero-frequency line broadening $(5-6 \mathrm{mT})$ and dc-resonance field shift $(50 \mathrm{mT})$ to a low field are both observed if the $\mathrm{Nb}$ thickness is comparable to the London penetration depth of $\mathrm{Nb}$ films ( $\geq 100 \mathrm{~nm}$ ). We attribute the observed peculiar behaviors to the increased incoherent precession near the $\mathrm{Ni}_{80} \mathrm{Fe}_{20} / \mathrm{Nb}$ interfaces and the effectively focused magnetic flux in the middle $\mathrm{Ni}_{80} \mathrm{Fe}_{20}$ caused by strong Meissner screening and (defect-)trapped flux of the thick adjacent $\mathrm{Nb}$ layers. This explanation is supported by static magnetic properties of the samples and comparison with FMR data on thick $\mathrm{Nb} / \mathrm{Ni}_{80} \mathrm{Fe}_{20}$ bilayers. Great care should, therefore, be taken in the analysis of FMR response in ferromagnetic Josephson structures with thick superconductors, a fundamental property for high-frequency device applications of spin-polarized supercurrents.
\end{abstract}

DOI: 10.1103/PhysRevApplied.11.014061

\section{INTRODUCTION}

In the past two decades, a ferromagnetic Josephson junction (FJJ) comprising two superconductors (SCs) separated by a ferromagnet (FM) has been of interest and developed extensively because of its unconventional physical properties [1-3] and potential applications in cryogenic computing technologies [4-10]. A very recent experimental work demonstrated that nanotextured FJJs integrated with standard single-flux quantum-neural systems form a new class of neuromorphic technologies that have spiking energies of less than $10^{-18} \mathrm{~J}$, operation frequencies up to $100 \mathrm{GHz}$, and nanoscale plasticity [11]. In particular, for an emergent field of superconducting spintronics [8-10], it has been recently established that the presence of a spatially varying magnetization $M(x)$ at $\mathrm{SC} / \mathrm{FM}$ interfaces can generate spin-polarized triplet supercurrents via spin mixing and spin rotation processes into the FM

*jeonkunrok@gmail.com
[12-14]. Interestingly, almost a decade ago, theoretical studies $[15,16]$ suggested a time-varying magnetization $M(t)$ of the FM as a reciprocal equivalent to $M(x)$ for the generation of spin-polarized triplet supercurrents in a diffusive metallic FJJ [15] and also in a FM/NM/SC structure (NM is normal metal) [9].

However, subsequent ferromagnetic resonance (FMR) studies on $\mathrm{Nb} / \mathrm{Ni}_{80} \mathrm{Fe}_{20}$ bilayers [17,18] and $\mathrm{Nb} / \mathrm{Ni}_{80} \mathrm{Fe}_{20} /$ $\mathrm{Nb}$ trilayers [19] have shown that spin-angular-momentum transfer in such structures is predominantly mediated by quasiparticles (QPs) for the superconducting state, and thus largely suppressed at a lower temperature $T$ by the development of singlet superconductivity and the freezeout of available QP states [17,20,21]. This is likely because the magnitude of $M(t)$ inhomogeneity or noncollinearity, parameterized by the magnetization precession angle $\theta_{M}$, is too small (a few degrees at 10-20 GHz) [17-19] to yield the measurable effect of $M(t)$-induced triplet supercurrents $[15,16]$. Another recent experiment, on the other hand, has reported that for $\mathrm{Ni}_{80} \mathrm{Fe}_{20}$ films sandwiched between 
rather thick $\mathrm{Nb}$ layers $(100 \mathrm{~nm})$ [22], the dc-resonance field shifts remarkably to a low field below the superconducting transition temperature $T_{c}$, interpreted as possible evidence for field-like spin-transfer torque (STT) induced by spin-triplet supercurrents.

According to the STT theory for metallic magnetic heterostructures [23-25], the antidamping STT is expected to be much larger (an order of magnitude) than the fieldlike STT due to the rapid dephasing of transverse spins in the FM [25]. It is thus of fundamental importance to test whether the effect of the antidamping torque (relevant to the Gilbert damping change $\Delta[\alpha]$ ) is consistent with that of the field-like torque (associated with the resonance field shift $\left.\Delta\left[\mu_{0} H_{\text {res }}\right]\right)$ in the superconducting state. Furthermore, knowledge about how magnetization dynamics of the FM changes by Meissner screening and magnetic flux pinning [26,27], especially in contact with thick SC layers, is highly desirable for the successful implementation of FMR functionality in FJJ-based superconducting spintronics [8-10]. Note that the triplet proximity channel, which is required to carry spin-angular momentum, depends on the strength of the underlying singlet superconductivity, and thus thicker superconducting electrodes are favorable for the generation of higher-density superconducting spin currents in FJJs [8-10,12-14].

Here, we focus on thick $\mathrm{Nb} / \mathrm{Ni}_{80} \mathrm{Fe}_{20} / \mathrm{Nb}$ trilayers where the $\mathrm{Nb}$ thickness $t_{\mathrm{Nb}}$ is comparable to the London penetration depth $\lambda_{L}$ of $\mathrm{Nb}$ films ( $\left.\geq 100 \mathrm{~nm}\right)$ [28] and so there exists a non-negligible effect of Meissner screening on the local (dc-rf) magnetic field experienced by the middle $\mathrm{Ni}_{80} \mathrm{Fe}_{20}$ layer. Through broadband (5-20 GHz) FMR measurements on such trilayers, we identify that the anomalous zero-frequency line broadening $\mu_{0} \Delta H_{0}$ and the significant $\Delta\left[\mu_{0} H_{\text {res }}\right]$ to a low field both appear below $T_{c}$. Importantly, the effect of $\Delta\left[\mu_{0} H_{\text {res }}\right]$ is found to be $1-2$ orders of magnitude larger than that of $\Delta[\alpha]$, which is incompatible with the STT theory [23-25]. We explain these peculiar behaviors in terms of locally perturbed magnetization precession of the middle $\mathrm{Ni}_{80} \mathrm{Fe}_{20}$ layer under spatially inhomogeneous magnetic fields caused by the strong Meissner effect and the magnetic flux pinning $[26,27]$ of the thick adjacent $\mathrm{Nb}$ layers. Static magnetic properties of the samples and comparison with FMR data on thick $\mathrm{Nb} / \mathrm{Ni}_{80} \mathrm{Fe}_{20}$ bilayers consistently support our explanation.

\section{EXPERIMENTAL DETAILS}

Polycrystalline $\mathrm{Nb} / \mathrm{Ni}_{80} \mathrm{Fe}_{20} / \mathrm{Nb}$ trilayers and $\mathrm{Nb} / \mathrm{Ni} i_{80}$ $\mathrm{Fe}_{20}$ bilayers are deposited on thermally oxidized Si substrates with lateral dimensions of $5 \times 5 \mathrm{~mm}^{2}$ using $\mathrm{dc}$ magnetron sputtering in an ultra-high vacuum chamber. The $\mathrm{Nb}\left(\mathrm{Ni}_{80} \mathrm{Fe}_{20}\right)$ thickness $t_{\mathrm{Nb}}\left(t_{\mathrm{Py}}\right)$ of $100(15) \mathrm{nm}$ is chosen to allow comparison with the recent FMR study on similar sample structures [22]. Details of the sample growth and $T_{c}$ characterization are described elsewhere [19].

We measure the FMR response of the sample attached on a broadband coplanar waveguide with either a dc field or rf pulse modulation [19]; to obtain each FMR spectrum, the microwave (MW) power absorbed by the sample is measured while sweeping the external static magnetic field $\mu_{0} \mathrm{H}$ at the fixed MW frequency $f$ of $5-20 \mathrm{GHz}$. Note that for all FMR measurements, the MW power is set to $10 \mathrm{dBm}$ where the actual MW power absorbed in the sample is a few $\mathrm{mW}$ that has no effect on $T_{c}$ of the $\mathrm{Nb}$ layer [19]. At the beginning of each measurement, we apply a large in-plane $\mu_{0} H(0.5 \mathrm{~T})$ to fully magnetize the $\mathrm{Ni}_{80} \mathrm{Fe}_{20}$ layer, after which the field is reduced to the range of FMR. Once the $f$ dependent FMR measurements (from high to low $f$ ) are complete, the field is returned to zero to cool the system down further for a lower $T$ measurement. We employ a vector field cryostat from Cryogenic Ltd. that can apply a 1.2-T-magnetic field in any direction over a $T$ range of 2-300 K. Some FMR measurements are conducted on the same samples using a different Helium flow cryostat from Oxford Instruments to test for reproducibility.

Magnetization properties of the same samples used for FMR measurements are characterized using a Quantum Design Magnetic Property Measurement System at $T$ varying between 2 and $300 \mathrm{~K}$. For all FMR and magnetization measurements, $\mu_{0} H$ is applied parallel to the film plane; a careful alignment of the film plane with respect to $\mu_{0} H$ is made to minimize any unintentional out-of-plane component of $\mu_{0} H$.

\section{RESULTS AND DISCUSSION}

\section{A. Temperature dependence of ferromagnetic resonance at different frequencies}

Let us first consider the $T$ evolution of FMR spectra for the $\mathrm{Nb}(100 \mathrm{~nm}) / \mathrm{Ni}_{80} \mathrm{Fe}_{20}(15 \mathrm{~nm}) / \mathrm{Nb}(100 \mathrm{~nm})$ trilayer. Figure 1(a) shows typical FMR data obtained at the two different $f$ of 10 and $20 \mathrm{GHz}$, from $80 \mathrm{~K}$ down to $2 \mathrm{~K}$. Note that all the FMR data presented are well fitted with the field derivative of symmetric and antisymmetric Lorentzian functions [29]. This enables us to accurately determine the FMR linewidth $\mu_{0} \Delta H$ (linked to the Gilbert damping $\alpha$ ) and the resonance field $\mu_{0} H_{\text {res }}$ (associated with the saturation magnetization $\mu_{0} M_{s}$ ). Overall $T$ dependences of $\mu_{0} \Delta H$ and $\mu_{0} H_{\text {res }}$ for various $f$ are summarized in Figs. 1(b) and 1(c), respectively. In the normal state $\left(T>T_{c}\right), \mu_{0} \Delta H$ and $\mu_{0} H_{\text {res }}$ are both almost independent of $T$. However, on entering the superconducting state $\left(T<T_{c}\right), \mu_{0} \Delta H$ broadens largely down to $4 \mathrm{~K}$ followed a slight fall at a lower $T$ and $\mu_{0} H_{\text {res }}$ shifts significantly to a low field; these effects are more pronounced for a lower $f$. This superconducting-state FMR response is quite different from that observed in the relatively thin $\mathrm{Nb} / \mathrm{Ni}_{80} \mathrm{Fe}_{20} / \mathrm{Nb}$ samples $\left(t_{\mathrm{Nb}} \leq 60 \mathrm{~nm} \ll \lambda_{L}\right)$ where $\Delta\left[\mu_{0} H_{\text {res }}\right]$ is less than 

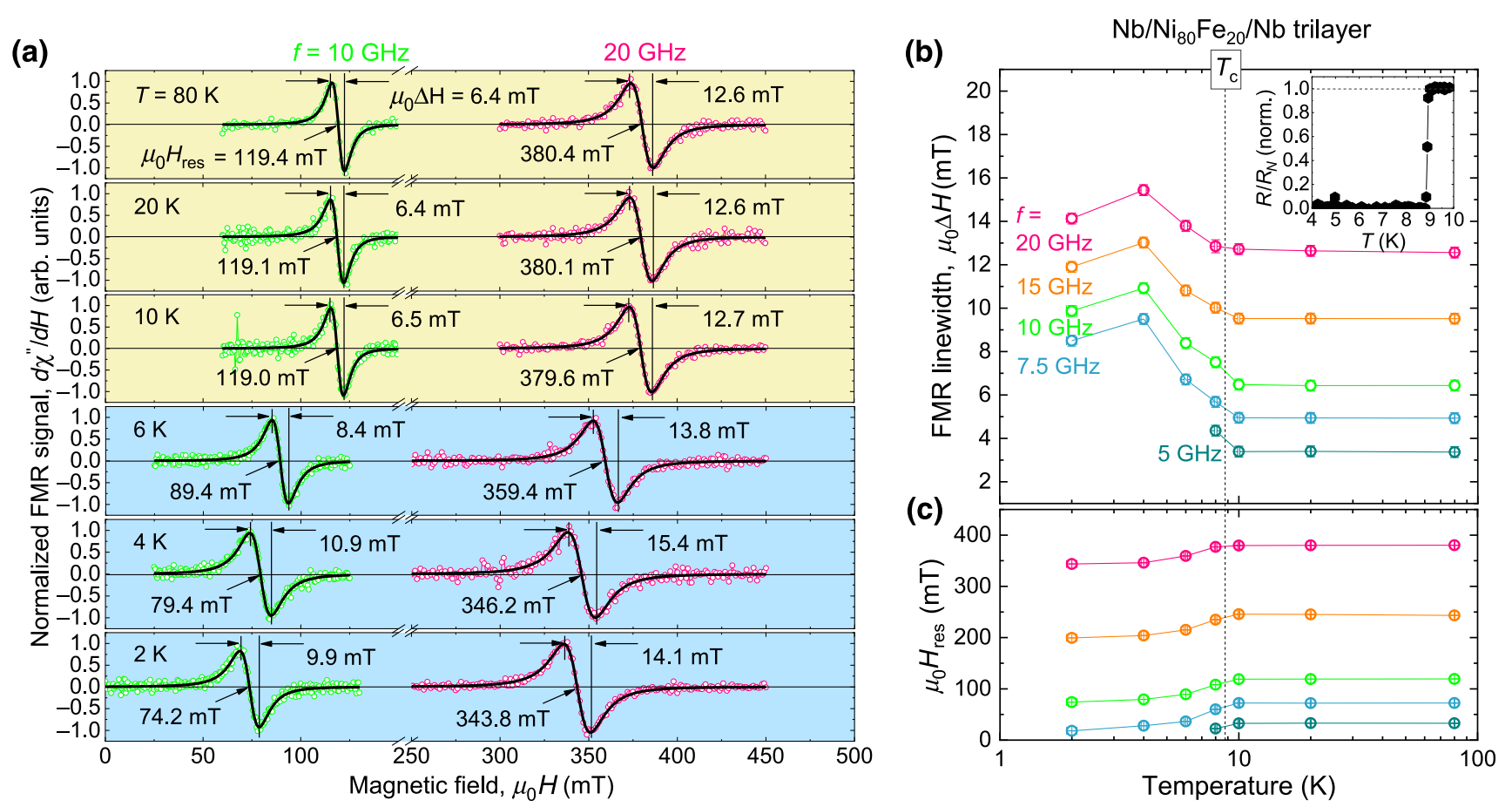

FIG. 1. (a) Typical FMR spectra for the thick $\mathrm{Nb}(100 \mathrm{~nm}) / \mathrm{Ni}_{80} \mathrm{Fe}_{20}(15 \mathrm{~nm}) / \mathrm{Nb}(100 \mathrm{~nm})$ trilayer obtained at the two different frequencies $f$ of 10 and $20 \mathrm{GHz}$, from $80 \mathrm{~K}$ down to $2 \mathrm{~K}$. The yellow (blue) background represents the normal (superconducting) state of $\mathrm{Nb}$. Temperature $T$ dependence of the FMR linewidth $\mu_{0} \Delta H$ (b) and the resonance magnetic field $\mu_{0} H_{\text {res }}$ (c) for the Nb/Ni $\mathrm{Ni}_{80} \mathrm{Fe}_{2} / \mathrm{Nb}$ trilayer. The inset shows the normalized resistance $R / R_{N}$ versus $T$ plot for the trilayer. Note that at $f=5 \mathrm{GHz}$ in (b), FMR signals become unmeasurable when $T<8 \mathrm{~K}$ as the amplitude of the resonance field shift to a low field goes beyond the typical resonance field (approximately $33 \mathrm{mT}$ ) in the normal state. It is also noteworthy that in (b), the peak point is determined by two competing effects of 1) Meissner screening and flux pinning and 2) spin-blocking behavior (see main text for details).

$2 \%$ (at $f=20 \mathrm{GHz}$ ) and $\mu_{0} \Delta H$ narrows monotonically below $T_{c}$ [19], implying that the $t_{\mathrm{Nb}}$-dependent superconductivity itself is responsible for the difference between them.

\section{B. Significant zero-frequency line broadening and resonance field shift below $T_{c}$}

For a quantitative analysis, we extract the Gilbert (-type) damping constant $\alpha$ from the linear scaling of $\mu_{0} \Delta H$ with $f$ at a fixed $T$ [Fig. 2(a)]; $\mu_{0} \Delta H(f)=$ $\mu_{0} \Delta H_{0}+4 \pi \alpha f / \sqrt{3} \gamma$ [30]. Here, $\mu_{0} \Delta H_{0}$ is the zerofrequency line broadening due to long-range magnetic inhomogeneities in the FM [31] and $\gamma$ is the gyromagnetic ratio $\left(1.84 \times 10^{11} \mathrm{~T}^{-1} \mathrm{~s}^{-1}\right)$ [32]. In the formula, we exclude other extrinsic broadening effects such as two-magnon scattering $[33,34]$ and Mosaicity broadening [34,35] as these have nonlinear $f$ and weak $T$ dependences. The extracted $\alpha$ [Fig. 2(b)] progressively decreases deep into the superconducting state $\left(T<T_{c}\right)$, which can be explained by the suppressed outflow of spin currents from the precessing $\mathrm{Ni}_{80} \mathrm{Fe}_{20}$ due to the development of singlet superconductivity in the adjacent $\mathrm{Nb}$ layers. This agrees with theoretical studies for proximity coupled metallic
FM/SC systems [18] and also with previous experiments [17,19-21].

In contrast, an anomalous $\mu_{0} \Delta H_{0}$ [inset of Fig. 2(b)] appears for the thick $\mathrm{Nb} / \mathrm{Ni}_{80} \mathrm{Fe}_{20} / \mathrm{Nb}$ sample $\left(t_{\mathrm{Nb}}=\right.$ $100 \mathrm{~nm}$ ) below $T_{c}$ and it reaches 5-6 mT at $2 \mathrm{~K}$, approximately an order of magnitude stronger than that in the relatively thin $\mathrm{Nb} / \mathrm{Ni}_{80} \mathrm{Fe}_{20} / \mathrm{Nb}$ samples $\left(t_{\mathrm{Nb}} \leq 60 \mathrm{~nm} \ll\right.$ $\lambda_{L}$ ) [19]. This implies that when $t_{\mathrm{Nb}}$ is comparable to $\lambda_{L}$ [28], the coupled superconducting $\mathrm{Nb}$ layers perturb local magnetization precession of the $\mathrm{Ni}_{80} \mathrm{Fe}_{20}$ layer and cause the incoherent precession near the $\mathrm{Ni}_{80} \mathrm{Fe}_{20} / \mathrm{Nb}$ interfaces.

The influence of superconductivity on $\mu_{0} H_{\text {res }}(f)$ can be described using the modified Kittel formula [36]: $f=$ $\gamma / 2 \pi \sqrt{\left[\mu_{0}\left(H_{\text {res }}+H_{\text {shift }}^{\mathrm{SC}}+M_{\text {eff }}\right) \cdot \mu_{0}\left(H_{\text {res }}+H_{\text {shift }}^{\mathrm{SC}}\right)\right]}$, where $\mu_{0} M_{\text {eff }}$ is the effective saturation magnetization and $\mu_{0} H_{\text {shift }}^{\mathrm{SC}}$ is the correction term that describes the superconductivity induced resonance field shift. In Fig. 2(c), we fit the $\mu_{0} H_{\text {res }}(f)$ data obtained at different (constant) $T$ using the Kittel formulas with and without the presence of $\mu_{0} H_{\text {shift }}^{\mathrm{SC}}$ for comparison. When $\mu_{0} H_{\text {shift }}^{\mathrm{SC}} \neq 0$, all the data are well fitted [Fig. 2(c)] and the estimated values of $\mu_{0} M_{\text {eff }}$ are in the range of 835-850 $\mathrm{mT}$ [Fig. 2(d)], which are similar to those obtained from static magnetometry measurements (Sec. III C). By contrast, when $\mu_{0} H_{\text {shift }}^{\mathrm{SC}}=0$, 
(a)

(c)
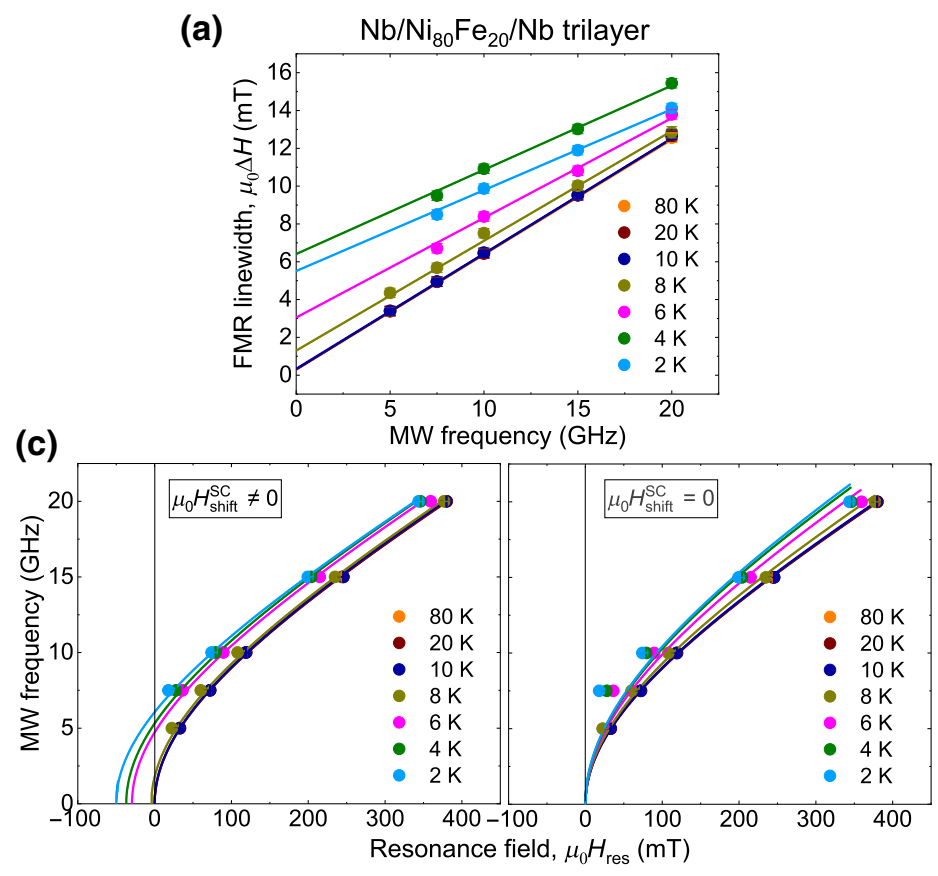

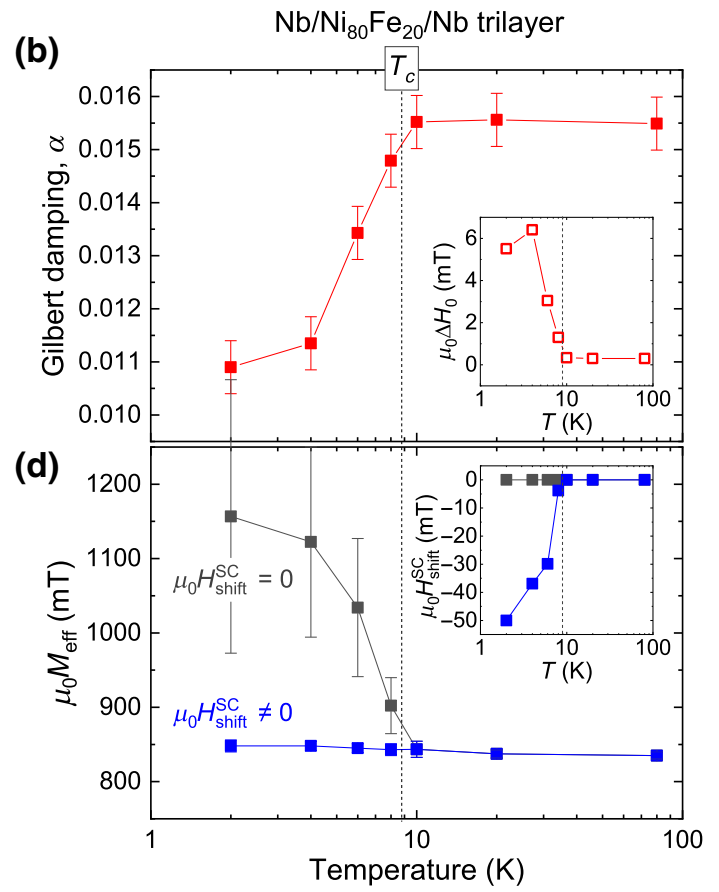

FIG. 2. (a) FMR linewidth $\mu_{0} \Delta H$ as a function of MW frequency for the $\mathrm{Nb}(100 \mathrm{~nm}) / \mathrm{Ni}_{80} \mathrm{Fe}_{20}(15 \mathrm{~nm}) / \mathrm{Nb}(100 \mathrm{~nm})$ trilayer at various temperatures $T$. The solid lines are linear fits to deduce the Gilbert damping constant $\alpha$ and the zero-frequency line broadening $\mu_{0} \Delta H_{0}$. (b) Deduced values of $\alpha$ and $\mu_{0} \Delta H_{0}$ (inset) as a function of $T$. (c) Microwave frequency vs resonance field $\mu_{0} H_{\text {res. }}$. The solid lines are fits to extract the effective saturation magnetization of the $\mathrm{Ni}_{80} \mathrm{Fe}_{20}$ layer using the modified Kittel formulas with (left) and without (right) the correction term $\mu_{0} H_{\text {shift }}^{\mathrm{SC}}$. (d) Extracted $\mu_{0} M_{\text {eff }}$ values (of the $\mathrm{Ni}_{80} \mathrm{Fe}_{20}$ ) vs $T$ with and without the presence of $\mu_{0} H_{\text {shift }}^{\mathrm{SC}}(T)$ (inset).

fitting the data gets worse at a lower $T$, in particular for a lower $f$ [Fig. 2(c)], and gives the anomalously increased $\mu_{0} M_{\text {eff }}$ below $T_{c}$ [Fig. 2(d)]. This points to the existence of an internal source of dc magnetic flux-field to the middle $\mathrm{Ni}_{80} \mathrm{Fe}_{20}$, accompanied by the onset of superconductivity in the $\mathrm{Nb}$ layers.

Notably, the extracted $\mu_{0} H_{\text {shift }}^{\mathrm{SC}}$ of $30-50 \mathrm{mT}$ [inset of Fig. 2(d)] is found to be 1-2 orders of magnitude larger than the FMR damping decrease of 0.0005-0.0045 for the superconducting state [Fig. 2(b)], corresponding to the $\mu_{0} \Delta H$ suppression of $0.4-3.5 \mathrm{mT}$ at $20 \mathrm{GHz}$ (in the dimension of the effective field). Since this result is inconsistent with the STT theory [23-25] described above, it is natural to consider an alternative explanation that is more relevant to the superconductivity-modified magnetization dynamics. The most common feature of SC films is the presence of Meissner screening and magnetic flux pinning [26,27].

\section{Static magnetic properties below $\boldsymbol{T}_{c}$}

To support our explanation of the FMR result, we perform static magnetometry measurements on the same samples (used for FMR measurements) across $T_{c}$. Figure 3(a) first shows the magnetization vs $T$ plots for the $\mathrm{Nb}(100 \mathrm{~nm}) / \mathrm{Ni}_{80} \mathrm{Fe}_{20}(15 \mathrm{~nm}) / \mathrm{Nb}(100 \mathrm{~nm})$ trilayer and the $\mathrm{Nb}(100 \mathrm{~nm}) / \mathrm{Ni}_{80} \mathrm{Fe}_{20}(15 \mathrm{~nm})$ bilayer. FMR data of the bilayer and their comparison with the trilayer will be presented below (Sec. III D). Above $T_{c}$ of the $\mathrm{Nb}$, the total magnetization $M_{\text {total }}$ of the sample is given solely by the ferromagnetic $\mathrm{Ni}_{80} \mathrm{Fe}_{20}$ layer, which is expected to increase weakly with decreasing $T$ (far below $T_{\text {Curie }}$ ) as $1-B T^{3 / 2}$ according to Bloch's law [37]. Here, $B$ is the Bloch constant or spin-wave parameter. A fair fit (black solid line) to the data [inset of Fig. 3(a)] is obtained with a reasonable $B$ of $1.25(1.26) \times 10^{-5} \mathrm{~K}^{-3 / 2}$ for the trilayer (bilayer), which is very close to the estimated value $\left(B=1.23 \times 10^{-5} \mathrm{~K}^{-3 / 2}\right)$ for bulk $\mathrm{Ni}_{80} \mathrm{Fe}_{20}$ [38]. On the other hand, below $T_{c}$, the Nb layers with type-II SC magnetization can contribute to $M_{\text {total }}$ (of the sample) in addition to the ferromagnetic $\mathrm{Ni}_{80} \mathrm{Fe}_{20}$. An abrupt change in $M_{\text {total }}$ (under the field cooling) when $T_{c}$ is crossed is indicative of this.

The $M_{\text {total }}$ vs in-plane $\mu_{0} H$ curves across $T_{c}$ are presented in Figs. 3(b) and 3(c) for the trilayer and the bilayer, respectively. Assuming that the superconducting state $M_{\text {total }}\left(\mu_{0} H\right)$ is a superposition of ferromagnetic $\mathrm{Ni}_{80} \mathrm{Fe}_{20}$ and $\mathrm{Nb}$ (type-II SC) magnetizations, one can separate the $\mathrm{Nb}$ magnetization $M_{\mathrm{Nb}}\left(\mu_{0} H\right)$ by taking the difference between the $M_{\text {total }}\left(\mu_{0} H\right)$ curves above and below $T_{c}$. We then get the characteristic type-II behavior in initial curves [insets of Figs. 3(b) and 3(c)]. The linear diamagnetic response to $\mu_{0} H$ (Meissner screening) is visible for 
(a)

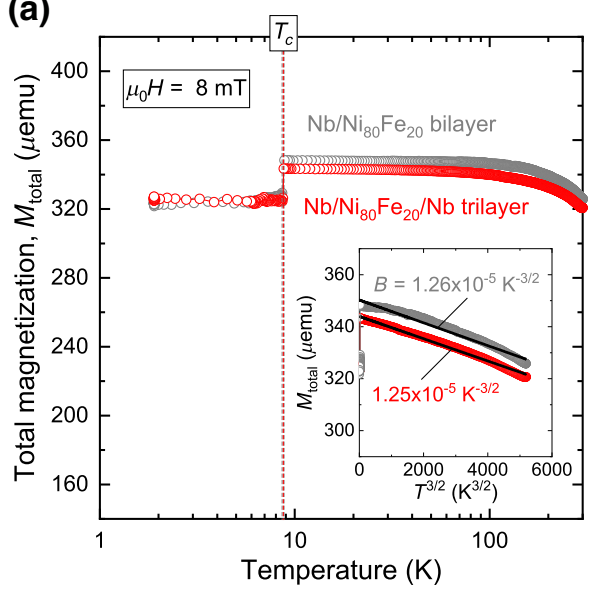

(b)

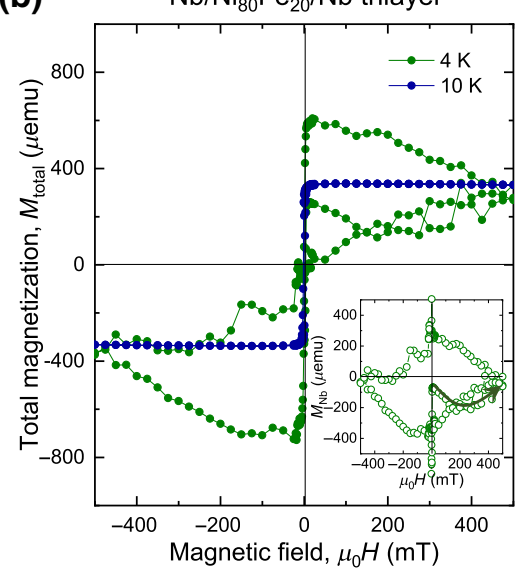

(c)

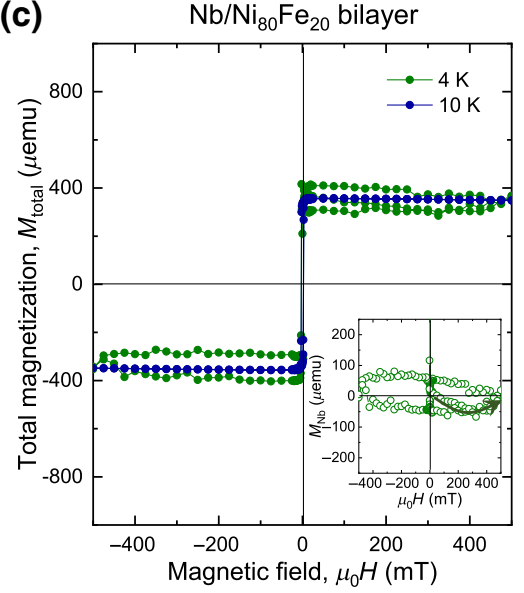

FIG. 3. (a) Total magnetization $M_{\text {total }}$ vs temperature $T$ plots for the $\mathrm{Nb}(100 \mathrm{~nm}) / \mathrm{Ni}_{80} \mathrm{Fe}_{20}(15 \mathrm{~nm}) / \mathrm{Nb}(100 \mathrm{~nm})$ trilayer and the $\mathrm{Nb}(100 \mathrm{~nm}) / \mathrm{Ni}_{80} \mathrm{Fe}_{20}(15 \mathrm{~nm})$ bilayer. $M_{\text {total }}(T)$ is attained while decreasing $T$ at the fixed/applied magnetic field $\mu_{0} H$ of $8 \mathrm{mT}$, which is far less than the lower critical field $\mu_{0} H_{c 1}$ of Nb layers. The $M_{\text {total }}$ vs (in-plane) magnetic field $\mu_{0} H$ curves, taken above and below the superconducting transition $T_{c}$ for the $\mathrm{Nb} / \mathrm{Ni}_{80} \mathrm{Fe}_{20} / \mathrm{Nb}$ trilayer (b) and the $\mathrm{Ni}_{80} \mathrm{Fe}_{20} / \mathrm{Nb}$ bilayer (c). The diamagnetic background signal from the quartz sample holder is subtracted. Each inset shows the isolated $\mathrm{Nb}$ magnetization $M_{\mathrm{Nb}}\left(\mu_{0} H\right)$ by taking the difference between the $M_{\text {total }}\left(\mu_{0} H\right)$ curves above and below $T_{c}$. The arrow in the inset is a guide to the eyes for the initial curve.

a small field range $(\leq 0.2 \mathrm{~T})$. After reaching an extremum at the lower critical field $\mu_{0} H_{c 1}$, the absolute magnetization drops as magnetic flux starts to penetrate the $\mathrm{Nb}$ until reaching the upper critical field $\mu_{0} H_{c 2}$ (a few Tesla for $\mathrm{Nb}$ films) $[19,39]$.

It is notable that magnetic flux pinning at defects in the $\mathrm{SC}$ can be inferred from the hysteresis behaviors, which emerge when $\mu_{0} H>\mu_{0} H_{c 1}$. The hysteresis area and the remaining magnetization at zero external field, quantifying the amount of flux pinning, are both expected to be much larger for thicker SCs where more defect sites and stronger Meissner screening co-exist [26,27]. The consistent behaviors seen in the $M_{\mathrm{Nb}}\left(\mu_{0} H\right)$ curves [insets of Figs. 3(b) and 3(c)] clarify that the non-negligible (a)

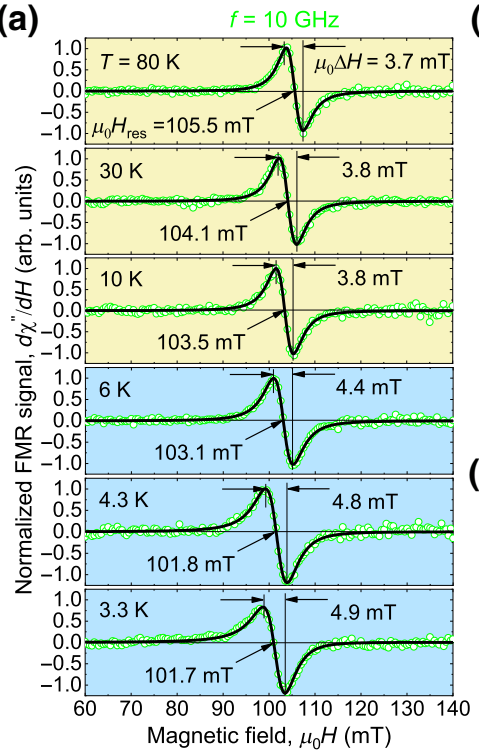

(b)

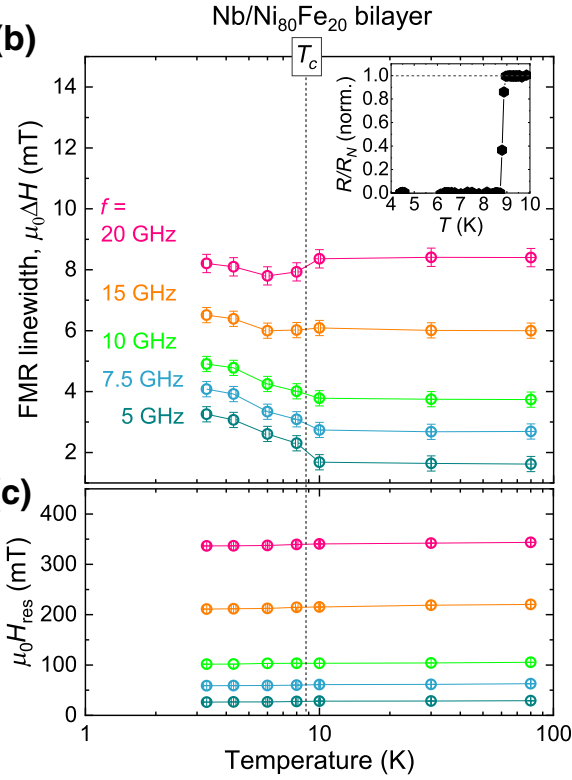

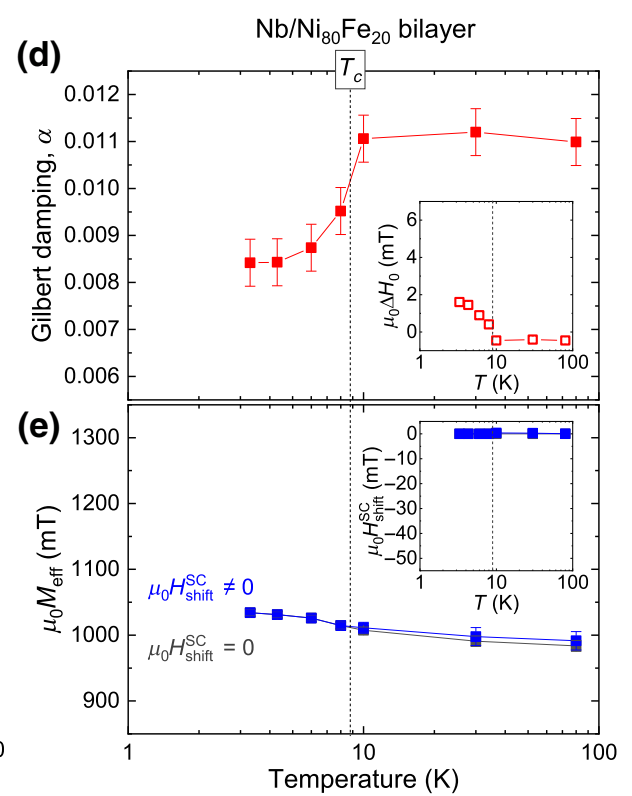

FIG. 4. (a) Representative FMR spectra for the thick $\mathrm{Nb}(100 \mathrm{~nm}) / \mathrm{Ni}_{80} \mathrm{Fe}_{20}(15 \mathrm{~nm})$ bilayer obtained at the fixed frequency $f$ of $10 \mathrm{GHz}$, from $80 \mathrm{~K}$ down to $2 \mathrm{~K}$. The yellow (blue) background represents the normal (superconducting) state of $\mathrm{Nb}$. Temperature $T$ dependence of the FMR linewidth $\mu_{0} \Delta H$ (b) and the resonance magnetic field $\mu_{0} H_{\text {res }}$ (c) for the $\mathrm{Nb} / \mathrm{Ni}_{80} \mathrm{Fe}_{2}$ bilayer. The inset exhibits the normalized resistance $R / R_{N}$ vs $T$ plot for the bilayer. (d) Estimated values of $\alpha$ and $\mu_{0} \Delta H_{0}$ (inset) as a function of $T$. (e) Extracted $\mu_{0} M_{\text {eff }}$ values (of the $\mathrm{Ni}_{80} \mathrm{Fe}_{20}$ ) vs $T$ with and without the presence of $\mu_{0} H_{\text {shift }}^{\mathrm{SC}}(T)$ (inset). Relevant details are presented in Appendix A. 
(defect-)trapped magnetic flux is present in the thick $\mathrm{Nb}$ samples.

In fact, the anomalous FMR response observed in the thick $\mathrm{Nb} / \mathrm{Ni}_{80} \mathrm{Fe}_{20} / \mathrm{Nb}$ trilayer below $T_{c}$ (Sec. III B) can be explained if we consider that the trapped magnetic flux at defects randomly distributed in the neighboring $\mathrm{Nb}$ layers serves as the internal source of additional magnetic field to the middle $\mathrm{Ni}_{80} \mathrm{Fe}_{20}$ under the external dc resonance field.

\section{Comparison with bilayers}

To further support our explanation, let us now discuss the FMR results (Fig. 4) taken from the $\mathrm{Nb}(100 \mathrm{~nm}) / \mathrm{Ni}_{80} \mathrm{Fe}_{20}(15 \mathrm{~nm})$ bilayer where overall flux pinning (of the sample) is weaker compared to the trilayer (Sec. III C). Figures 4(a)-4(c) show that for the bilayer, the change of FMR spectra, that is, $\mu_{0} \Delta H$ and $\mu_{0} H_{\text {res }}$, as a function of $T$ below $T_{c}$ is indeed weaker than for the trilayer. Note that as $\mu_{0} \Delta H$ tends to increase at a lower $T$ [Fig. 4(b)], it results in the occurrence of superconductivity induced line broadening, as discussed below.

For better understanding and quantitative comparison, we plot the $T$ dependences of $\alpha, \mu_{0} H_{0}, \mu_{0} M_{\text {eff }}$, and $\mu_{0} H_{\text {shift }}^{\mathrm{SC}}$ values in Figs. 4(d) and 4(e), extracted from the $f$ dependent FMR data (see Appendix A for details). It is worth noting that the quantitative change of $\alpha$ reduction (or spin-pumping damping) across $T_{c}$ [Fig. 4(d)] is approximately twice weaker relative to the trilayer [Fig. 2(b)], which is in accordance with spin pumping through a single $\mathrm{Nb} / \mathrm{Ni}_{80} \mathrm{Fe}_{20}$ interface $[17,18]$ and thereby is a single spin-blocking effect of the $\mathrm{Nb}$ layer [17-21].

Perhaps the most noteworthy aspect of the bilayer data is that even though the resonance field shift is very small $[<|0.5| \mathrm{mT}$, inset of Fig. 4(e)], as in the previous experiment [16], there still exists the anomalous increase of $\mu_{0} H_{0}$ at a lower $T$ [+2 $\mathrm{mT}$ at $3.3 \mathrm{~K}$, inset of Fig. 4(d)] that is approximately three times smaller than the trilayer [inset of Fig. 2(b)], but large enough to compensate the FMR linewidth suppression $[-2.5 \mathrm{mT}$ at $3.3 \mathrm{~K}$ at $20 \mathrm{GHz}$, Fig. 4(d)] resulting from the aforementioned spin-blocking effect [17-21]. It, in turn, makes the $T$ dependence of total linewidth nontrivial [Fig. 4(b)], highlighting that broadband FMR measurements are of utmost importance for proper interpretation of the experimental results.

The bilayer result suggests that the FMR linewidth change is more sensitive than the resonance field shift to the local flux pinning and so the $f$ dependent linewidth analysis may be useful to somehow isolate the genuine spin-triplet proximity effect [8-10] from other extrinsic phenomena $[40,41]$ being driven in $\mathrm{FM} / \mathrm{SC}$ interfaces, a key ingredient for developing superconducting spintronics.

\section{CONCLUSIONS}

How Meissner screening and (defect-)trapped magnetic flux affect magnetization dynamics in thick
$\mathrm{Nb} / \mathrm{Ni}_{80} \mathrm{Fe}_{20} / \mathrm{Nb}$ trilayers is investigated by using broadband FMR spectroscopy. We find that when $t_{\mathrm{Nb}}$ is comparable to $\lambda_{L}$ of $\mathrm{Nb}$ films, anomalous $\mu_{0} \Delta H_{0}$ and significant $\Delta\left[\mu_{0} H_{\text {res }}\right]$ to a low field both appear below $T_{c}$. Notably, the effect of $\Delta\left[\mu_{0} H_{\text {res }}\right]$ is found to be much greater than that of $\Delta[\alpha]$ in the superconducting state, which is incompatible with the STT theory. We consider the superconductivity modified magnetization dynamics as an alternative explanation for the FMR data, which is convincingly supported by static magnetic properties of the samples and comparison with FMR data on thick $\mathrm{Nb} / \mathrm{Ni}_{80} \mathrm{Fe}_{20}$ bilayers. Our results suggest that careful consideration should be made when analyzing FMR data in FJJs with thick SCs. Proper selection of SC properties provides a pathway to dynamically access the spin-polarized supercurrents in SC/FM proximity coupled systems [8-10] for their potentials in high-frequency device applications.

\section{ACKNOWLEDGMENTS}

This work was supported by EPSRC Programme Grant No. EP/N017242/1.

\section{APPENDIX A: ANALYSIS OF FREQUENCY DEPENDENCE OF FERROMAGNETIC RESONANCE SPECTRA FOR THE BILAYER}

Using the same approach as for the trilayer (Sec. III B), we extract the $T$ dependences of $\alpha, \mu_{0} H_{0}, \mu_{0} M_{\text {eff }}$, and $\mu_{0} H_{\text {shift }}^{\text {SC }}$ values [presented in Figs. 4(d) and 4(e)] from the $f$ dependent FMR data acquired on the thick $\mathrm{Nb}(100 \mathrm{~nm}) / \mathrm{Ni}_{80} \mathrm{Fe}_{20}(15 \mathrm{~nm})$ bilayer (Fig. 5). Note that there exists a visible increase of $\mu_{0} H_{0}$ (zero-frequency intercept) at a lower $T$ [Fig. 5(a)] even though the resonance field shift is tiny [Fig. 5(b)].

\section{APPENDIX B: DISCUSSION OF MEISSNER SCREENING EFFECT ON MAGNETIC DOMAIN STRUCTURE}

It was previously reported that for disk-patterned$\mathrm{Al}(150 \mathrm{~nm}) / \mathrm{Ni}(50 \mathrm{~nm})$ samples of submicron size in zero external field [42], Meissner screening of the domain's stray fields by the adjacent Al layer can cause a spatial redistribution of magnetic domains and its $T$ dependence is connected with the screening capability of the Al. Even if this mechanism would, in principle, increase magnetization inhomogeneity below $T_{c}$, it is unclear that this could be used to explain our results, where continuous samples are used in FMR study under application of a large external field $(30-400 \mathrm{mT})$. In this vein, it is of interest to systematically investigate how the superconducting-state FMR response is altered by changing the type of FM (having a different strength of stray fields) in $\mathrm{FM} / \mathrm{SC}$ bilayer and $\mathrm{SC} / \mathrm{FM} / \mathrm{SC}$ trilayer structures. 
(a)
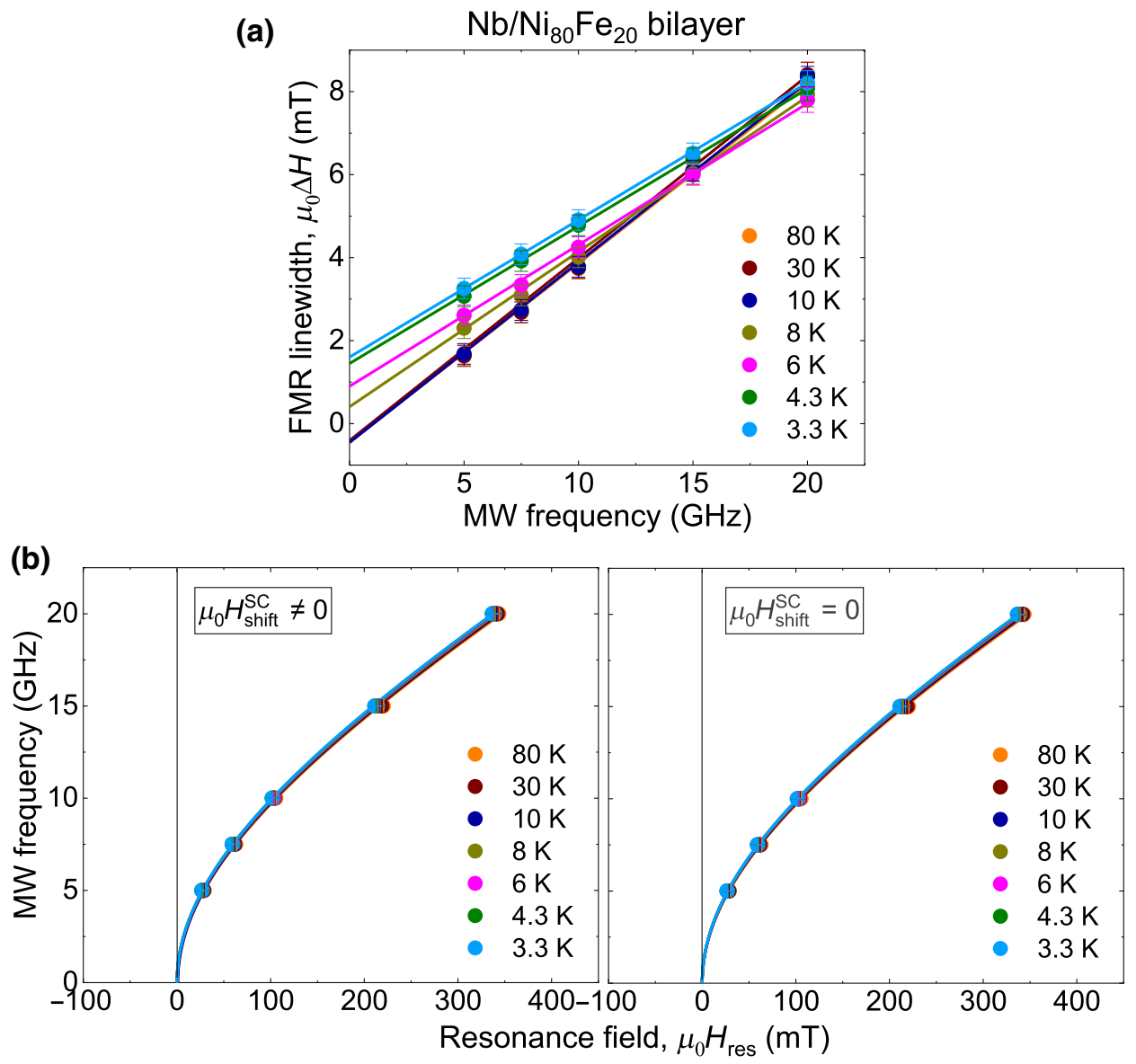

FIG. 5. Data equivalent to Figs. 2(a) and 2(c), but for the $\mathrm{Nb}(100 \mathrm{~nm}) / \mathrm{Ni}_{80} \mathrm{Fe}_{20}(15 \mathrm{~nm})$ bilayer.

[1] T. Kontos, M. Aprili, J. Lesueur, F. Genêt, B. Stephanidis, and R. Boursier, Josephson Junction through a Thin Ferromagnetic Layer: Negative Coupling, Phys. Rev. Lett. 89, 137007 (2002).

[2] J. W. A. Robinson, S. Piano, G. Burnell, C. Bell, and M. G. Blamire, Zero to $\pi$ transition in superconductorferromagnet-superconductor junctions, Phys. Rev. B 76, 094522 (2007).

[3] S. Piano, J. W. A. Robinson, G. Burnell, and M. G. Blamire, $0-\pi$ oscillations in nanostructured $\mathrm{Nb} / \mathrm{Fe} / \mathrm{Nb}$ Josephson junctions, Eur. Phys. J. B 58, 123 (2007).

[4] A. A. Golubov, M. Yu. Kupriyanov, and E. Il'ichev, The current-phase relation in Josephson junctions, Rev. Mod. Phys. 76, 411 (2004).

[5] A. I. Buzdin, Proximity effects in superconductorferromagnet heterostructures, Rev. Mod. Phys. 77, 935 (2005).

[6] F. S. Bergeret, A. F. Volkov, and K. B. Efetov, Odd triplet superconductivity and related phenomena in superconductor-ferromagnet structures, Rev. Mod. Phys. 77, 1321 (2005).

[7] M. Houzet and A. I. Buzdin, Long range triplet Josephson effect through a ferromagnetic trilayer, Phys. Rev. B 76, 060504(R) (2007).
[8] J. Linder and J. W. A. Robinson, Superconducting spintronics, Nat. Phys. 11, 307 (2015).

[9] M. Eschrig, Spin-polarized supercurrents for spintronics: a review of current progress, Rep. Prog. Phys. 78, 104501 (2015).

[10] N. O. Birge, Spin-triplet supercurrents, Phil. Trans. R. Soc. A 376, 20150150 (2018).

[11] M. L. Schneider, C. A. Donnelly, S. E. Russek, B. Baek, M. R. Pufall, P. F. Hopkins, P. D. Dresselhaus, S. P. Benz, and W. H. Rip, Ultralow power artificial synapses using nanotextured magnetic Josephson junctions, Sci. Adv. 4, e1701329 (2018).

[12] J. W. A. Robinson, J. D. S. Witt, and M. G. Blamire, Controlled injection of spin-triplet supercurrents into a strong ferromagnet, Science 329, 59 (2010).

[13] T. S. Khaire, M. A. Khasawneh, W. P. Pratt, Jr., and N. O. Birge, Observation of Spin-Triplet Superconductivity in Co-Based Josephson Junctions, Phys. Rev. Lett. 104, 137002 (2010).

[14] J. W. A. Robinson, F. Chiodi, M. Egilmez, G. B. Halász, and M. G. Blamire, Supercurrent enhancement in Bloch domain walls, Sci. Rep. 2, 699 (2012).

[15] M. Houzet, Ferromagnetic Josephson Junction with Precessing Magnetization, Phys. Rev. Lett. 101, 057009 (2008).

[16] T. Yokoyama and Y. Tserkovnyak, Tuning odd triplet superconductivity by spin pumping, Phys. Rev. B 80, 104416 (2009). 
[17] C. Bell, S. Milikisyants, M. Huber, and J. Aarts, Spin Dynamics in a Superconductor-Ferromagnet Proximity System, Phys. Rev. Lett. 100, 047002 (2008).

[18] J. P. Morten, A. Brataas, G. E. W. Bauer, W. Belzig, and Y. Tserkovnyak, Proximity-effect-assisted decay of spin currents in superconductors, Eur. Phys. Lett. 84, 57008 (2008).

[19] K.-R. Jeon, C. Ciccarelli, A. J. Ferguson, H. Kurebayashi, L. F. Cohen, X. Montiel, M. Eschrig, J. W. A. Robinson, and M. G. Blamire, Enhanced spin pumping into superconductors provides evidence for superconducting pure spin currents, Nat. Mater. 7, 499 (2018).

[20] J. Y. Gu, J. A. Caballero, R. D. Slater, R. Loloee, and W. P. Pratt, Direct measurement of quasiparticle evanescent waves in a dirty superconductor, Phys. Rev. B 66, 140507(R) (2002).

[21] T. Wakamura, N. Hasegawa, K. Ohnishi, Y. Niimi, and Y. Otani, Spin Injection into a Superconductor with Strong Spin-Orbit Coupling, Phys. Rev. Lett. 112, 036602 (2014).

[22] L.-L. Li, Y.-L. Zhao, X.-X. Zhang, and Y. Sun, Possible evidence for spin-transfer torque induced by spin-triplet supercurrents, Chin. Phys. Lett. 35, 077401 (2018).

[23] A. Brataas, A. D. Kent, and H. Ohno, Current-induced torques in magnetic materials, Nat. Mater. 11, 372 (2012).

[24] K. Xia, P. J. Kelly, G. E. W. Bauer, A. Brataas, and I. Turek, Spin torques in ferromagnetic/normal-metal structures, Phys. Rev. B 65, 220401 (2002).

[25] M. A. Zimmler, B. Özyilmaz, W. Chen, A. D. Kent, J. Z. Sun, M. J. Rooks, and R. H. Koch, Current-induced effective magnetic fields in $\mathrm{Co} / \mathrm{Cu} / \mathrm{Co}$ Nanopillars, Phys. Rev. B 70, 184438 (2004).

[26] S. Aull, O. Kugeler, and J. Knobloch, Trapped magnetic flux in superconducting niobium samples, Phys. Rev. STAB. 15, 062001 (2012).

[27] R. Huebener, Magnetic Flux Structures in Superconductors, edited by M. Cardona, P. Fulde, K. v. Klitzing, H.-J. Queisser, R. Merlin, and H. Störmer, Springer Series in Solid-State Science Vol. 6 (Springer, New York, 2001), 2nd ed., p. 311.

[28] A. I. Gubin, K. S. Il'in, and S. A. Vitusevich, Dependence of magnetic penetration depth on the thickness of superconducting $\mathrm{Nb}$ thin films, Phys. Rev. B 72, 064503 (2005).

[29] Z. Celinski, K. B. Urquhart, and B. Heinrich, Using ferromagnetic resonance to measure the magnetic moments of ultrathin films, J. Magn. Magn. Mater. 166, 6 (1997).

[30] K. Ando, S. Takahashi, J. Ieda, Y. Kajiwara, H. Nakayama, T. Yoshino, K. Harii, Y. Fujikawa, M. Matsuo, S. Maekawa, and E. Saitoh, Inverse spin-Hall effect induced by spin pumping in metallic system, J. Appl. Phys. 109, 103913 (2011).

[31] B. Heinrich, Ultrathin Magnetic Structures, Vol. III (Springer, Berlin, 2005).

[32] J. M. Shaw, H. T. Nembach, T. J. Silva, and C. T. Boone, Precise determination of the spectroscopic g-factor using broadband ferromagnetic resonance spectroscopy, J. Appl. Phys. 114, 243906 (2013).

[33] Kh. Zakeri, J. Lindner, I. Barsukov, R. Meckenstock, M. Farle, U. von Hörsten, H. Wende, W. Keune, J. Rocker, S. S. Kalarickal, K. Lenz, W. Kuch, K. Baberschke, and Z. Frait, Spin dynamics in ferromagnets: Gilbert damping and two-magnon scattering, Phys. Rev. B 76, 104416 (2007).

[34] K. Lenz, H. Wende, W. Kuch, K. Baberschke, K. Nagy, and A. Jánossy, Two-magnon scattering and viscous Gilbert damping in ultrathin ferromagnets, Phys. Rev. B 73, 144424 (2006).

[35] S. Mizukami, Y. Ando, and T. Miyazaki, The study on ferromagnetic resonance linewidth for $\mathrm{NM} / 80 \mathrm{NiFe} / \mathrm{NM}$ $(\mathrm{NM}=\mathrm{Cu}, \mathrm{Ta}, \mathrm{Pd}$ and $\mathrm{Pt}$ ) films $>$, Jpn. J. Appl. Phys. 40, 580 (2001).

[36] C. Kittel, On the theory of ferromagnetic resonance absorption, Phys. Rev. 73, 155 (1948).

[37] W. Kipferl, M. Dumm, M. Rahm, and G. Bayreuther, Thermal spin excitations in epitaxial Fe nanostructures on GaAs(001), J. Appl. Phys. 93, 7601 (2003).

[38] D. Scholl, M. Donath, D. Mauri, E. Kay, J. Mathon, R. B. Muniz, and H. C. Siegmannb, Exchange interactions at the surface of a ferromagnet, Phys. Rev. B 43, 13309 (1991).

[39] C. Cirillol, C. Voltan, E. A. Ilyina, J. M. Hernández, A. García-Santiago, A. Aarts, and C. Attanasio, Long-range proximity effect in $\mathrm{Nb}$-based heterostructures induced by a magnetically inhomogeneous permalloy layer, New J. Phys. 19, 023037 (2017).

[40] Mark Rubinstein, P. Lubitz, W. E. Carlos, P. R. Broussard, D. B. Chrisey, J. Horwitz, and J. J. Krebs, Properties of superconductor-ferromagnet bilayers: $\mathrm{YBa}_{2} \mathrm{CuO}_{3}-\mathrm{Fe}$ and $\mathrm{YBa}_{2} \mathrm{CuO}_{3}$-Permalloy, Phys. Rev. B 47, 11535 (1993).

[41] I. A. Golovchanskiy, N. N. Abramov, V. S. Stolyarov, V. V. Bolginov, V. V. Ryazanov, A. A. Golubov, and A. A. Ustinov, Ferromagnet/superconductor hybridization for magnonic applications, Adv. Funct. Mater. 28, 1802375 (2018).

[42] S. V. Dubonos, A. K. Geim, K. S. Novoselov, and I. V. Grigorieva, Spontaneous magnetization changes and nonlocal effects in mesoscopic ferromagnet-superconductor structures, Phys. Rev. B 65, 220513(R) (2002). 